\title{
Disruption of White Matter Integrity in Bipolar Depression as a Possible Structural Marker of Illness
}

\author{
Francesco Benedetti, Ping-Hong Yeh, Marcella Bellani, Daniele Radaelli, Mark A. Nicoletti, Sara Poletti, \\ Andrea Falini, Sara Dallaspezia, Cristina Colombo, Giuseppe Scotti, Enrico Smeraldi, Jair C. Soares, and \\ Paolo Brambilla
}

Background: Diffusion tensor imaging allows the study of integrity of white matter (WM) tracts. Literature suggests that WM integrity could be altered in bipolar disorder. Heterogeneity of brain imaging methods, the studied samples, and drug treatments make localization, nature, and severity of the WM abnormalities unclear.

Methods: We applied tract-based spatial statistics of diffusion tensor imaging measures to compare fractional anisotropy (FA), mean, and radial diffusivity of the WM skeleton in a group of 40 consecutively admitted inpatients affected by a major depressive episode without psychotic features with a diagnosis of bipolar disorder type I and 21 unrelated healthy volunteers from the general population.

Results: Compared with control subjects, patients showed lower FA in the genu of the corpus callosum and in anterior and right superior-posterior corona radiata and higher values of radial diffusivity in WM tracts of splenium, genu and body of corpus callosum, right mid-dorsal part of the cingulum bundle, left anterior and bilateral superior and posterior corona radiata, bilateral superior longitudinal fasciculus, and right posterior thalamic radiation. Patients had no brain areas with higher FA or lower diffusivity values than control subjects.

Conclusions: Reduced FA with increased mean and radial diffusivity suggests significant demyelination and/or dysmyelination without axonal loss. Comparing our findings with other observations in homogeneous samples of euthymic and manic patients, it can be hypothesized that changes in measures of WM integrity might parallel illness phases of bipolar illness.

Key Words: Bipolar disorder, cingulum, corona radiata, corpus callosum, diffusion tensor imaging, MRI methodology

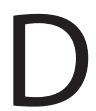

iffusion tensor imaging (DTI) assesses the microscopic diffusion of water and allows the indirect investigation of the integrity and bundle coherence of brain white matter (WM) tracts. Given the microscopic structure of WM, in normal conditions the integrity of myelinated axons limits the diffusion of water in directions other than along the axis of the fiber. This tendency to diffuse in one direction as opposed to all others, termed anisotropy, can be estimated through the application of diffusion-sensitizing gradients and the calculation of elements of the diffusion tensor matrix, i.e., the three eigenvalues $\lambda_{1}, \lambda_{2}$, and $\lambda_{3}(1-3)$.

Fractional anisotropy (FA) values range between 0 , when water motion is random in all directions, and 1, when the directional

From the Department of Clinical Neurosciences (FB, DR, SP, SD, CC, ES), Scientific Institute and University Vita-Salute San Raffaele; and Centro di Eccellenza Risonanza Magnetica ad Alto Campo (FB, DR, SP, AF, SD, CC, GS, ES), Milano, Italy; Department of Psychiatry (P-HY, PB), University of North Carollina, Chapel Hill, North Carolina; Inter-University Center for Behavioural Neurosciences, Department of Medicine and Public Health, Section of Psychiatry and Clinical Psychology (MB), University of Verona, Verona; and Department of Pathology and Experimental \& Clinical Medicine, Section of Psychiatry (PB), University of Udine, Udine, Italy; Department of Psychiatry and Behavioral Sciences (MAN, JCS), University of Texas-Houston Medical School, Houston, Texas; and Dottorato in Neuroscienze e Disturbi del Comportamento (SP), Università degli Studi di Palermo, Palermo; Department of Neuroradiology (AF, GS), Scientific Institute and University Vita-Salute San Raffaele, Milano; and Scientific Institute (PB), Istituto di Ricovero e Cura a Carattere Scientifico Eugenio Medea, Udine, Italy.

Address correspondence to Francesco Benedetti, M.D., Istituto Scientifico Ospedale San Raffaele, Department of Clinical Neurosciences, San Raffaele Turro, Via Stamira d'Ancona 20, 20127 Milano, Italy; E-mail: benedetti. francesco@hsr.it.

Received Mar 15, 2010; revised Jul 27, 2010; accepted Jul 29, 2010. selectivity of water motion is maximal. Fractional anisotropy can reflect the structure of axonal cell membranes and myelin sheaths (4). A decrease of the tendency to diffuse along the principal direction $\left(\lambda_{1}\right)$ of the fiber, axial diffusivity, suggests axonal loss or loss of bundle coherence, and an increase in radial diffusivity (RD) (the average of $\lambda_{2}$ and $\lambda_{3}$ ), perpendicular to axonal walls, suggests disrupted myelination (5). Mean diffusivity (MD) (average of $\lambda_{1}, \lambda_{2}$, and $\lambda_{3}$ ) is a measure of the average molecular motion, independent of tissue directionality. In WM pathologies involving disruption of myelin sheaths, the reduction of FA is usually paralleled by an increase of MD (6). High FA values are observed in heavily myelinated tracts and decline with age, and a reduction of FA is observed along the progression of several degenerative WM disorders that result in axonal loss or destruction of myelin sheaths, such as multiple sclerosis, leukoaraiosis, various dementias, and infections (6).

Several lines of evidence, coming both from neuropsychological studies and from functional and structural brain imaging reports, suggest that disrupted neural connectivity and brain network dysfunction are core biological correlates of bipolar disorder and could play a role in the mechanistic explanation of its symptomatology (7). Disrupted connectivity might be due to WM pathology, as suggested by the observation of an increased number of hyperintense WM lesions at T2-weighted magnetic resonance imaging scanning (8), a signal enhanced where degradation of the myelin sheath leads to local edema $(4,9)$.

Focusing on the a priori hypothesized brain regions, the DTI studies using region of interest approach reported reduced $F A$ with normal apparent diffusion coefficients in adult patients in the regions of superior frontal WM $(n=9)(10)$, reduced FA in anteriormiddle corpus callosum ( $n=37$ ) (11) and anterior cingulum $(n=$ 42) (12), and reduced FA in the anterior limb of the internal capsule, anterior thalamic radiation, and in the uncinate fasciculus $(n=42)$ $(13,14)$. However, increased diffusivity $(n=8)(15)$ and normal FA $(n=14)$ (16) were also reported. Moreover, even increased FA in anterior frontal regions, with reduced $F A$ in fronto-occipital fasciculi $(n=33)(17)$, increased FA in corpus callosum $(n=11)(18)$, and 
normal FA and MD in several regions together with more axonal fibers and increased connectivity between subgenual cingulate and the amygdala $(n=16)(19)$ were observed.

Extending the DTI study to the whole brain using voxelwise analysis, there was reported decreased FA in temporo-occipital regions, with increased MD in frontal and prefrontal WM $(n=36)$ (20) and reduced FA in the genu of corpus callosum, right inferior longitudinal fasciculus, and left superior longitudinal fasciculus $(n=19)$ (21). Significantly decreased FA and increased MD in bilateral prefrontal-limbic-striatal white matter and right inferior frontooccipital, superior, and inferior longitudinal fasciculi were observed in currently depressed $(n=16)$ but not in currently euthymic ( $n=$ 21) patients (22). However, higher FA in bilateral frontal white matter with lower FA in the left cerebellar white matter has also been reported $(n=30)(23)$.

Recent studies used tract-based spatial statistics (TBSS), which focuses on the centers of all fiber bundles that are common to the participants (the most compact WM skeleton), thus improving the probability that the given space voxels contain data from the same part of the same WM tract among each participant (24). Using this approach in bipolar patients experiencing heterogeneous states of the illness (depression, mania, or euthymia) and treated with multiple combined drugs $(n=31)$, abnormally high left FA and low right $F A$, respectively coupled with lower or higher radial diffusivity, were found in orbitomedial prefrontal regions (25). The most recent study, however, reported a diffusely higher FA in medial frontal, precentral, inferior, parietal, and occipital white matter of euthymic patients $(n=22)(26)$

Considering FA, the above cited literature reported it to be decreased in studies involving 159 cases, normal in 51, and increased in 33 , with three studies in 94 cases reporting lower or higher values in different brain regions in the same groups. Although confirming the interest for the DTI study of WM integrity in bipolar disorder, these inconsistencies clearly raised the need for further studies taking into account the whole WM compartment and the psychopathological status of the patients. Remarkably, the recently developed TBSS, which appears to potentially overcome the problems linked with smoothing and WM tracts alignment of other wholebrain methods (24), gave opposite results in the samples studied up to now. The localization, the nature, and the severity of the WM abnormalities associated with bipolar illness remained unclear. Nonetheless, if chronic mood homeostasis is one of the core features of bipolar disorder, then it is conceivable that both hyperdense or hypodense white matter intensities or fasciculi could result in deficits in neuropsychiatric system level deficits (27). This might explain how diverse white matter endophenotypes can all result in bipolar disorder, because both heightened and reduced fractional anisotropy of fasciculi could result in concomitant changes in effective connectivity and dysregulation of higher order neural control systems.

To determine precisely the extent and the quality, if any, of the WM abnormalities linked with bipolar disorder, here we studied the WM integrity by means of TBSS in a unique homogeneous sample of 40 adult patients affected by a major depressive episode in the course of bipolar disorder, drug-free or treated with lithium alone.

\section{Methods and Materials}

\section{Participants}

The sample included 61 participants. We studied 40 inpatients selected from among 347 patients affected by mood disorders consecutively admitted to our ward in Milano. Inclusion criteria were to be affected by a major depressive episode, without psy- chotic features, with a diagnosis of bipolar disorder type I (Structured Clinical Interview for DSM Disorders), and to be either drugfree $(n=26)$ or have been on treatment with lithium alone $(n=14)$ for at least 6 months. Exclusion criteria were additional diagnoses on Axis I, mental retardation on Axis II, pregnancy, major medical and neurological disorders, or history of drug or alcohol abuse or dependency. No patient had received electroconvulsive therapy within 6 months before study enrollment. Physical examination, laboratory tests, and electrocardiograms were performed at admission. Severity of depression was rated on the 21-item Hamilton Depression Rating Scale (HDRS). Twenty-one healthy subjects with no previous history of psychiatric, neurological, and systemic disorders served as control subjects. After complete description of the study to the participants, written informed consent was obtained. The study was approved by the local ethical committee.

\section{Image Acquisition}

Diffusion tensor imaging was performed on a 3.0 Tesla scanner (Gyroscan Intera, Philips, Best, The Netherlands) using spin-echo echo-planar imaging and the following parameters: repetition time/ echo time $=8753.89 / 58 \mathrm{msec} ; 55$ contiguous, $2.3-\mathrm{mm}$ thick axial slices with field of view $=240 \mathrm{~mm}$; matrix scan 112; reconstructed 128 ; acquired voxel measure, phase and size $(\mathrm{mm})=2.14 / 2.71 / 2.30$; reconstructed voxel measure, phase and size $(\mathrm{mm})=1.88 / 1.87 / 2.30$, sensitivity encoding acceleration factor $=2,35$ noncollinear directions of the diffusion gradients, $\mathrm{b}$ value $=900 \mathrm{sec} / \mathrm{mm}^{2}$. Fat saturation was performed to avoid chemical shift artifacts.

On the same occasion and using the same magnet 22 Turbo Spin Echo (Philips), T2 axial slices (repetition time $=3000 \mathrm{msec}$; echo time $=85 \mathrm{msec}$; flip angle $=90^{\circ}$; turbo factor $15 ; 5$-mm-thick, axial slices with a $512 \times 512$ matrix and a $230 \times 230 \mathrm{~mm}^{2}$ field of view) were acquired to rule out brain lesions.

\section{Data Processing and Analysis}

The diffusion-weighted images were preprocessed by aligning to the nondiffusion-weighted image $(b=0)$ using affine registration for eddy current and motion correction (28). Simple least squares fit of the DTI model, FA, MD, parallel diffusivity, and RD were calculated. Tract based spatial statistics were performed. All FA images were nonlinearly co-registered to a FA template (FMRIB58-FA, FMRIB Centre University of Oxford, Department of Clinical Neurology, John Radcliffe Hospital Headington, Oxford, United Kingdom; http://www.fmrib.ox.ac.uk/ fsl/tbss/FMRIB58_FA.html) and normalized to the Montreal Neurological Institute space.

The perpendicular direction of the local surface tract was estimated and the highest FA along this direction was identified as the center of the tract (skeleton) to create the mean FA image and its skeleton. The entire study sample was used to create the mean FA skeleton. All individual FA values were then projected onto the mean FA skeleton. The maximum FA value was searched and then assigned to the current skeleton voxel. Voxelwise statistical analyses were performed on the skeletonized FA, MD, parallel diffusivity, and RD images, where FA $>.2$.

After accounting for age and gender effects, we executed a permutation-based nonparametric inference within the framework of the general linear model to investigate the differences between the three groups of healthy control subjects and patients with or without ongoing lithium treatment. Threshold-free cluster enhancement (TFCE) was performed, a permutation-based approach tested the voxel $t$ value, and TFCE estimated cluster size for the familywise error correction of multiple comparisons (corrected $p<$ $.05,5000$ permutations). Threshold-free cluster enhancement can be seen as a generalization of the cluster mass statistics (29) and 
Table 1. Clinical and Demographic Characteristics of the Sample Divided According to Diagnosis and Treatment

\begin{tabular}{|c|c|c|c|c|c|c|}
\hline & $\begin{array}{l}\text { Control Subjects } \\
\quad(n=21)\end{array}$ & $\begin{array}{l}\text { Drug-Free Patients } \\
\quad(n=26)\end{array}$ & $\begin{array}{l}\text { Lithium-Treated Patients } \\
\qquad(n=14)\end{array}$ & $F$ or $\chi^{2}$ & $d f$ & $p$ \\
\hline Age (Years) & $39.86 \pm 11.05$ & $45.11 \pm 9.82$ & $47.79 \pm 13.26$ & 2.10 & 2,58 & .131 \\
\hline Gender (Males/Females) & $11 / 10$ & $5 / 9$ & $5 / 21$ & 5.67 & 2 & .059 \\
\hline Age at Onset (Years) & - & $31.58 \pm 10.12$ & $31.14 \pm 7.78$ & .21 & 2,38 & .647 \\
\hline Duration of Illness (Years) & - & $13.54 \pm 9.95$ & $17.64 \pm 10.62$ & 1.48 & 2,38 & .231 \\
\hline Depressive Episodes $(n)$ & - & $5.27 \pm 5.59$ & $4.07 \pm 2.81$ & .56 & 2,38 & .458 \\
\hline Manic Episodes ( $n$ ) & - & $3.19 \pm 4.89$ & $2.57 \pm 2.06$ & .20 & 2,38 & .654 \\
\hline HDRS Score & - & $20.12 \pm 8.85$ & $19.14 \pm 6.27$ & .13 & 2,38 & .718 \\
\hline
\end{tabular}

Age at onset: Age at which the patient first met criteria for either major depression or mania. Data are means \pm standard deviations.

HDRS, Hamilton Depression Rating Scale.

uses spatial neighborhood information in a nonlinear image processing to increase sensitivity and boost the height of spatially distributed signals without changing the location of their maxima. Voxelwise levels of significance, corrected for multiple comparisons, are then calculated with a standard permutation testing by building up the null distribution (across permutation of the input data) of the maximum (across voxels) TFCE scores and then using the 95th percentile of the null distribution to threshold signals at corrected $p<.05$ (30). We evaluated whether the voxelwise diffusion parameters derived from the participants affected by bipolar disorder correlated with the HDRS scores after accounting for the effects of age and gender. We used Randomize (Department of Biostatistics, University of Michigan, Ann Arbor, Michigan) (31), a permutation program enabling modeling and inference using standard general linear model design setup. For each contrast, an effective regressor is formed using the original full design matrix and the contrast, as well as a new set of effective confound regressors, which are then preremoved from the data. Statistical inference $p$ values were corrected using TFCE after permutation testing.

\section{Results}

Clinical and demographic characteristics of participants are provided in Table 1. No difference was statistically significant.

Average values ofDTI measures and localization of areas with maximal differences between patients and control subjects are listed in Table 2.

Table 2. Values of Fractional Anisotropy and Radial and Mean Diffusivity of Control Subjects and Patients (Mean \pm Standard Deviation) in All the Clusters Showing Significant Differences Between Groups and Regions Showing Maximal Differences of TBSS Values (Signal Peaks) Between Patients and Control Subjects

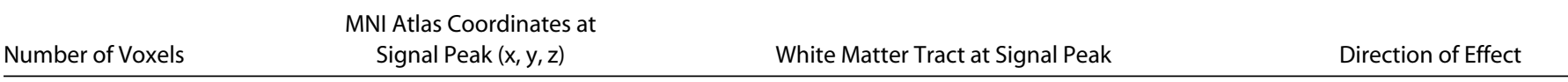

All Patients Compared with Control Subjects

Fractional Anisotropy (control subjects: .619 \pm .143 ; patients: .586 \pm .145 )

$\begin{array}{lll}1260 & 1230-6 & \text { Genu of corpus callosum } \\ 506 & 16-4039 & \text { Right dorsal cingulum }\end{array}$

Radial Diffusivity (control subjects: .465 \pm .084 : patients: $.492 \pm .086$ ) $16550 \quad 23-4238$

Mean Diffusivity (control subjects: .679 \pm .063 ; patients: .703 \pm .067 )

$13182 \quad 22-4226$
Right posterior corona radiata

Splenium of corpus callosum/ posterior corona radiata
Control subjects $>$ patients Control subjects $>$ patients

Patients $>$ control subjects

Patients $>$ control subjects

Drug-Free Patients Compared with Control Subjects

Radial Diffusivity (control subjects: .471 \pm .073 : patients: $.502 \pm .076$ )

$\begin{array}{ll}2759 & 16-4240 \\ 85 & 37-222\end{array}$

Mean Diffusivity (control subjects: $.720 \pm .055$; patients: $.703 \pm .067$ )

$253 \quad 16-4126$

$65 \quad 7-2723$
Right dorsal cingulum

Right superior longitudinal fasciculus

Splenium of corpus callosum

Body of corpus callosum
Lithium-Treated Patients Compared with Control Subjects

Fractional Anisotropy (control subjects: $.557 \pm .148$; patients: $.526 \pm .146$ )

$\begin{array}{ll}909 & 16340 \\ 840 & 25-3042 \\ 309 & -103010 \\ 171 & -21-4040\end{array}$

Radial Diffusivity (control subjects: .464 \pm .086 : patients: $.489 \pm .087$ )

$13514 \quad 25-3142$

Mean Diffusivity (control subjects: .692 \pm .057 ; patients: .719 \pm .062 )

$\begin{array}{ll}1718 & 19-3536 \\ 65 & 31-689\end{array}$

Right superior longitudinal fasciculus

Patients $>$ control subjects

Patients $>$ control subjects

Patients $>$ control subjects

Patients $>$ control subjects

MNI, Montreal Neurological Institute; TBSS, tract-based spatial statistics.
Right anterior corona radiata

Right superior longitudinal fasciculus

Genu of corpus callosum

Left posterior corona radiata

Control subjects $>$ patients Control subjects $>$ patients Control subjects $>$ patients Control subjects $>$ patients

Patients $>$ control subjects

Right posterior corona radiata Right posterior thalamic radiation
Patients $>$ control subjects Patients $>$ control subjects 


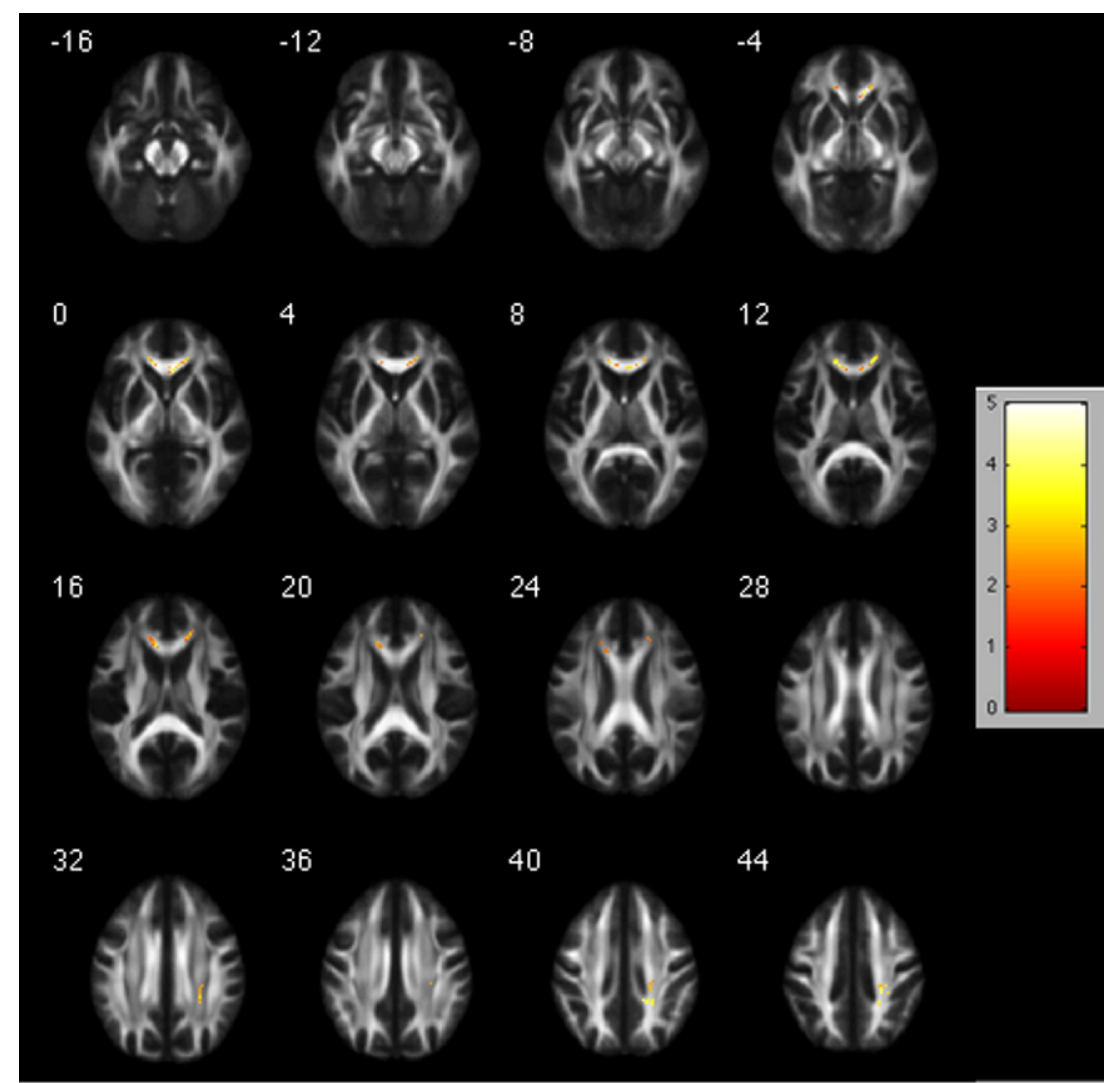

Figure 1. White matter areas where patients had significantly lower values of fractional anisotropy than control subjects. The color bar refers to $t$ values for the observed differences. Group differences are mapped onto the standard Montreal Neurological Institute atlas MNI152 1-mm brain template. Numbers are $\mathrm{z}$ coordinates.
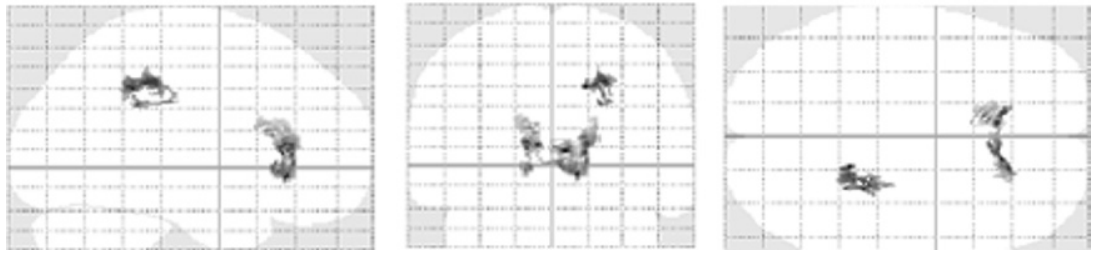

Compared with control subjects, patients showed significantly lower FA and higher $\mathrm{MD}$ and $\mathrm{RD}$ in multiple brain regions, while no difference in parallel diffusivity was observed. In no region did patients show higher FA or lower RD and MD than control subjects.

In detail, bipolar depressed patients as a group had lower FA in WM tracts of the genu of corpus callosum, bilateral anterior corona radiata, and in right superior and posterior corona radiata (Figure 1). Patients showed higher RD in a large cluster, including WM tracts of splenium, genu, and body of corpus callosum; right middorsal part of the cingulum bundle; left anterior and bilateral superior and posterior corona radiata; bilateral superior longitudinal fasciculi; and right posterior thalamic radiation including optic radiation (Figure 2). Finally, patients showed larger MD values in the splenium, genu, and body of corpus callosum; right middorsal part of cingulum bundle; right anterior and bilateral superior and posterior corona radiata; right superior longitudinal fasciculus; and right posterior thalamic radiation (Figure 3 ).

A post hoc power calculation (32) showed that, despite the statistical significance of the between-group effect in the abovementioned regions, the effect size of the average differences between patients and control subjects was small (Cohen's $d=.229$ for $\mathrm{FA}, \mathrm{d}=.369$ for $\mathrm{RD}, \mathrm{d}=.318$ for $\mathrm{MD}$ ), thus leading to a low power (respectively, .21, .39, and .32).

When comparing the three groups (healthy control subjects and patients with or without ongoing lithium treatment), we observed the following (Table 2): 1) patients with lithium did not significantly differ from unmedicated patients on any DTI measure; 2) patients with lithium significantly differed from control subjects, showing lower FA and higher MD and RD; and 3) patients without lithium significantly differed from control subjects, showing higher RD and MD but not different FA.

The HDRS scores were not found significantly correlated with DTI measures after controlling age effect and correcting for multiple comparisons.

\section{Discussion}

We observed reduced FA and increased mean and radial diffusivity, with conserved parallel diffusivity, in multiple brain regions of a sample of patients affected by a major depressive episode in the course of bipolar disorder. The distribution of abnormalities suggests a more severe disruption of WM integrity in cingulum, corpus callosum, and corona radiata, where lower FA values were coupled with higher mean and radial diffusivity (Table 2). Given that an increase of radial diffusivity is thought to signify increased space between fibers, suggesting demyelination or dysmyelination, and a decrease in parallel diffusivity suggests axonal injury (5), this finding suggests disrupted integrity of myelin sheaths without axonal loss. Other abnormal findings of increased radial and mean diffusivity, but no change of FA, were distributed across different regions, 


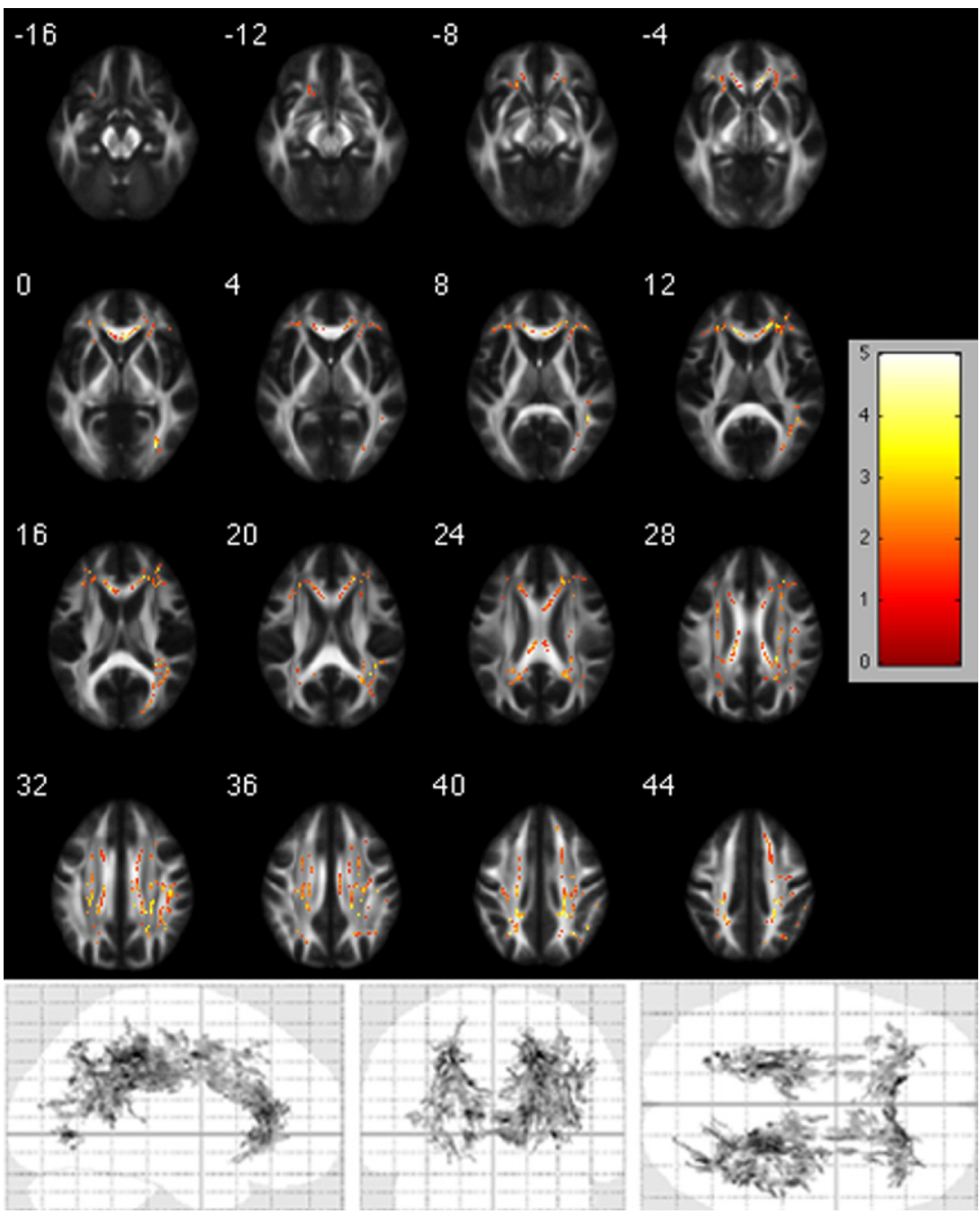

Figure 2. White matter areas where patients had significantly higher values of radial diffusivity than control subjects. The color bar refers to $t$ values for the observed differences. Group differences are mapped onto the standard Montreal Neurological Institute atlas MNI152 1-mm brain template. Numbers are z coordinates.

particularly in the right hemisphere (including cingulum bundle, superior longitudinal fasciculus, and thalamic radiation), thus suggesting less severe disruption of WM integrity (Figures 1-3).

These localizations include key WM tracts contributing to the functional integrity of the brain. Compromised communication involving interhemispheric connections and limbic and large frontal, parietal, and fronto-occipital connections could be a major biological underpinning of the hypothesized brain network dysfunctions leading to the cognitive and emotional deficits typical of bipolar depression and could contribute to explain the abnormal effective corticolimbic connectivity observed with functional magnetic resonance imaging in bipolar patients $(33,34)$. Corpus callosum integrity is crucial for sustained attention and context processing, which are frequently impaired in bipolar patients (35). Compromised white matter tract integrity of connections involving cortical and subcortical structures of the anterior limbic network (7) and anterior prefrontal network (36) are consistent with the concept of disrupted neurocircuitry involved in impulse control and emotional regulation, which are core features of bipolar disorder (see a comprehensive review in [37].

This partially confirms previous reports in the field about reduced FA in bipolar disorder (see above). In contrast with the two previous studies using TBSS $(25,26)$, in no brain regions did we observe an increased FA or a reduced diffusivity. Several nonalternative hypotheses could explain these discrepancies. In particular, the previous TBSS observations of a diffuse FA increase in euthymic patients treated with heterogeneous drugs (26) and of increased left with decreased right FA in patients with heterogeneous illness phases and mixed drug treatment (25) could either be due to the effect of drugs and/or to state-dependent changes in WM integrity measures.

The essential feature of bipolar disorder is a clinical course that is characterized by recurrent mood episodes of different polarity (depressive, manic, or mixed) alternating with euthymia. Euthymic and pathological states can last for years. The intriguing question whether DTI abnormalities associated with bipolar disorder are state- or trait-related requires longitudinal studies to be answered, but it can be hypothesized that changes in WM integrity might parallel the dramatic clinical changes across illness phases. A survey of the literature about DTI in adult bipolar patients shows that in several studies the current state of the patient was not reported $(10,20,23)$ or the sample included patients with heterogeneous clinical states $(11,12,16,25,38)$. These latter studies observed either reduction of FA $(11,12,16,38)$ or mixed abnormalities depending on the studied brain region (25). Five studies have been performed on euthymic $(18,22,26)$ or relatively stable $(13,17)$ patients, and three of them observed an increased FA in patients with bipolar disorder compared with control subjects. Previous to our own study in nonpsychotic bipolar depression, a pilot study in eight inpatients affected by bipolar depression with psychotic symptoms reported an 


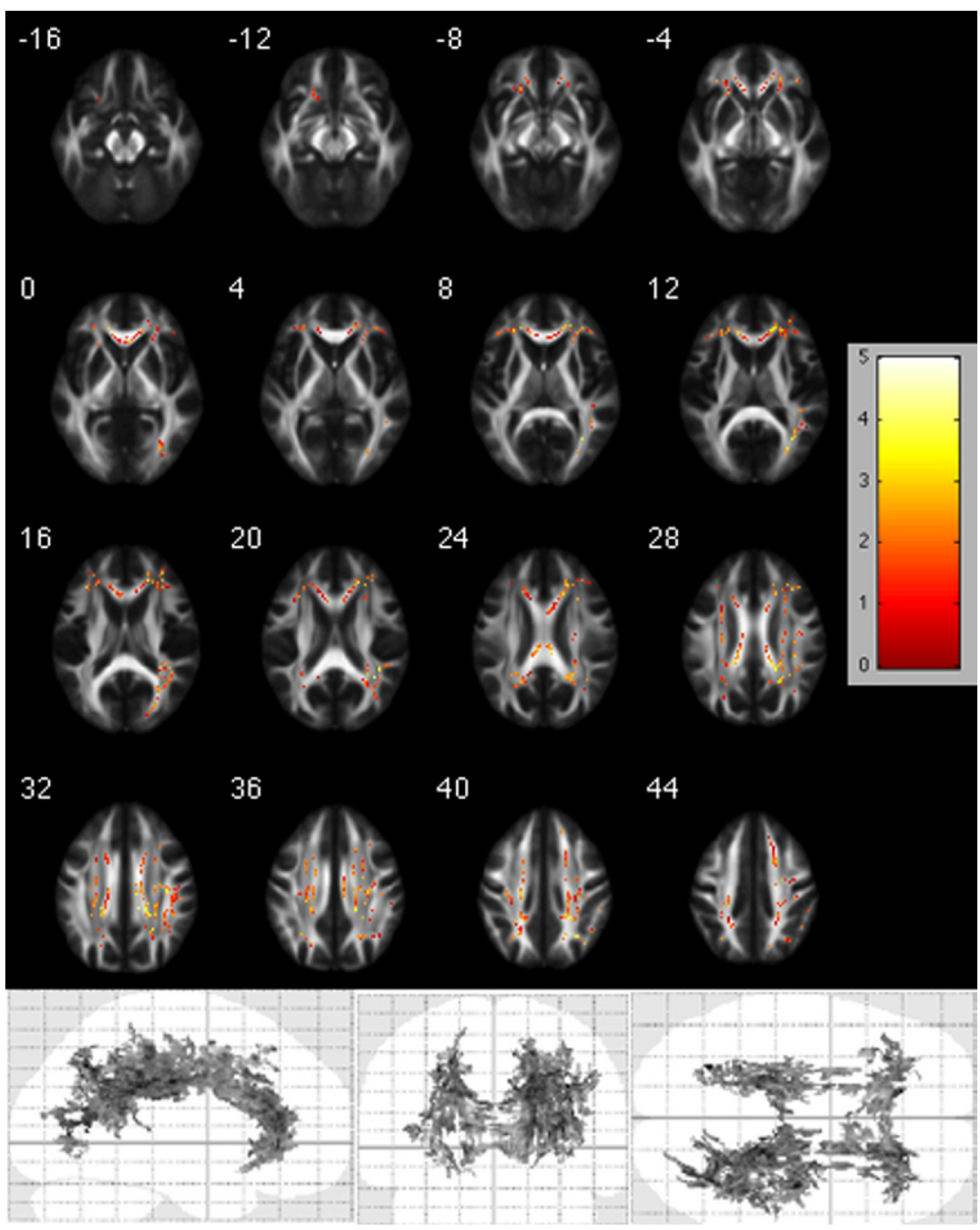

Figure 3. White matter areas where patients had significantly higher values of mean diffusivity than control subjects. The color bar refers to $t$ values for the observed differences. Group differences are mapped onto the standard Montreal Neurological Institute atlas MNI152 1-mm brain template. Numbers are $z$ coordinates.

increased diffusivity in frontal, temporal, and occipital region of interest (15); a study in patients with subsyndromal symptoms (Beck Depression Inventory score $7.9 \pm 7.0$ [mean \pm SD]) observed a reduced FA in several brain regions including the genu of the corpus callosum and right inferior and left superior longitudinal fasciculi (21); and a study in patients with heterogeneous drug treatments confirmed a diffusely reduced FA (22).

It is tempting to speculate that critical bipolar illness phases could be associated with DTI findings suggesting disrupted WM integrity (22) (reduced FA and increased radial and mean diffusivity), with opposite observations in stable euthymia (increased FA, increased connectivity [19]). In agreement with this hypothesis, in a mixed sample, currently depressed patients had lower FA values than remitted patients (25), while among clinically stable patients, subsyndromal depression ratings correlated inversely with FA (13). Following this perspective, it should be noted that the increase of FA in euthymic conditions could well reflect neuroplasticity (26) but that several factors such as increases in myelination, microscopic deficits of axonal structures, or decreases in axonal diameter, packing density, and fiber branching may cause higher directionality and contribute to higher FA in the regions where fibers cross, which has been associated with neuropsychological deficits in some neurological conditions (39). Persistent WM abnormalities associated with euthymic intervals could parallel persistent neuropsychological deficits in bipolar disorder (40).
Notwithstanding statistical significance, the differences observed by us were subtle, with low effect sizes (lowest for FA, highest for $\mathrm{RD}$ and $\mathrm{MD}$ ) resulting in low achieved power. This leaves the issue of lithium effects on WM integrity open, because it hampers the interpretation of the differences observed when separately comparing lithium-treated and lithium-free patients (who did not differ among themsleves) with healthy control subjects, when lithium-free patients showed abnormal RD and MD but not FA. Lithium can increase gray matter volumes, and two longitudinal studies recently associated its therapeutic efficacy in bipolar disorder with this effect $(41,42)$. Lithium could also improve WM integrity: it affects myelin gene expression (43), promotes structural and functional recovery in spinal cord-lesioned rats (44), and in humans it diffusely increased FA and decreased MD values in the brain of patients with human immunodeficiency virus infection, thus leading to better functional and metabolic patterns (45). Contradicting the above, a specific toxic effect of lithium in our patients could contribute to explain abnormal FA in lithium-treated but not in lithium-free patients. Alternative explanations, in agreement with the literature, include type II error due to insufficient power of the stratified sample and/or possible higher severity of illness, different previous drug treatments, and different biological substrates in patients who had chosen to take long-term lithium medication. Direct comparisons between lithium-treated and unmedicated patients were negative in our dataset, including areas where both 
groups significantly differed from control subjects, and Table 2 shows that the localization of peak differences and the dimensions of the clusters were different. Only prospective studies similar to those performed on gray matter, and far beyond the aim of the present one, can clarify this issue by testing the effects of lithium in unmedicated patients, who showed, however, abnormally high RD and MD. While disrupted integrity of myelin sheaths consistently leads to increased RD with preserved axial diffusivity, changes of FA can be due to a number of factors affecting local diffusivity, such as bundle coherence, crossing fibers, or water concentration (46-48). This could have contributed to differences in effect sizes for the different measures and also supports altered WM integrity unconfounded by current drug treatment in our lithium-free patients.

In a similar way, a low variability of HDRS scores among patients who were currently hospitalized for depression could have hampered the detection of a relationship between this measure and TBSS values.

Aging reduces WM integrity, as detected by using TBSS analysis on DTI (4), but the few studies in young patients with bipolar disorder confirmed WM abnormalities. In children, reduced FA was reported in corpus callosum, orbitofrontal cortex, and bilateral superior frontal tracts, including cingulate and paracingulate regions (49), and in anterior corona radiata (50) of patients with heterogeneous clinical states. In adolescents suffering a manic episode, reduced FA with normal apparent diffusion in superior frontal WM (51) and lower FA in the right orbital frontal lobe, with higher diffusivity in the right and left subgenual regions (52), were reported, and lower FA values in the fornix, cingulate gyrus, corpus callosum, and parietal and occipital corona radiata, with normal diffusivity, were observed in adolescent patients with mixed clinical states (38).

The pattern of abnormal findings described by us has been observed in neurological conditions associated with inflammation, degeneration, demyelination, or dysmyelination (53). An altered myelination during development, resulting in abnormal findings early in the pediatric age, has been proposed to play a role in the pathophysiology of bipolar illness (54). A single study in patients affected by bipolar disorder and their relatives observed a significant correlation between reduced FA across distributed regions of white matter and a quantitative measure of genetic liability for the disorder (21), but in young healthy adults, reduced FA has been associated with higher adverse childhood experiences due to harsh parenting (55), thus suggesting major gene-environment interactions. Postmortem gene expression, neuropathological, and neuroimaging studies suggested downregulation of key oligodendrocyte and myelination genes (56), as well as lowered density of oligodendroglial cells (57). Several findings support the notion of an altered immune function specific to bipolar illness (58), which could affect myelin maintenance and repair and play a role in the pathophysiology of the disease $(35,59)$.

All these mechanisms, and others to be hypothesized, could contribute to disrupt the integrity of WM. The question as to whether white matter integrity in bipolar disorder is a function of state-dependent processes (e.g., loss of myelination because of disease processes occurring over weeks or months) or occurs because of inherited traits cannot in principle be answered by a crosssectional study, and only a longitudinal or perhaps cross-sequential design can answer this question. In this respect, it should be noted that the only disorder that showed strong state-dependent changes in myelination occurring over weeks was multiple sclerosis. Longitudinal studies, performed in the same centers with the same methods, will also protect against possible arbitrary DTI methodological choices. In this respect, it should be noted that TBSS measures of WM integrity have been correlated with both functional connectivity among brain areas in schizophrenia (60) and with increasing neuropsychological performance during normal adolescence (61), thus suggesting that these DTI indexes likely reflect the multiple biological processes that occur during brain development and psychopathological processes that provide the neural substrate for ongoing functional connectivity.

Limitations of the present study, which is correlational in nature, also include issues such as generalizability, possible population stratification, a different sex ratio among groups, concomitant medications and their effects on the observed differences, nondrug naïve, no placebo control, no evaluation for compliance, varying treatment periods, no consideration of gene-environment interactions, and technical issues such as slice thickness that could limit the specificity of the regional differences or specific issues related to TBSS, which limits the study of the WM tracts that are out of the centers of the fiber bundles (the WM skeleton).

The Centro di Eccellenza Risonanza Magnetica ad Alto Campo received research grants from the Italian Ministry of University and Scientific Research, the Italian Ministry of Health, the European Union (FP7 Grant 222963), Trenta ore per la Vita Association, and JanssenCilag.

$F B$ designed the study. ES, FB, GS, JCS, and PB obtained the funding. $C C, S D, A F, F B$, and $E S$ were involved in participants' recruitment and selection. $D R, S P$, and $S D$ carried out the brain imaging scanning and collected the clinical data with the supervision of FB. P-HY designed the tract-based spatial statistics-threshold-free cluster enhancement data analyses and carried them out with contributions from MAN and MB. $F B$ wrote the first draft of the manuscript, with other authors contributing to data interpretation and final manuscript preparation. All authors take final responsibility for the decision to submit for publication. $F B$ and P-HY had full access to all of the data in the study and take responsibility for the integrity of the data and the accuracy of the data analysis.

JCS reports consulting/speaking for Lilly, AstraZeneca, and Pfizer, and received research grants from Lilly, Repligen, GlaxoSmithKline, and Pfizer. All other authors report no biomedical financial interests or potential conflicts of interest.

1. Le Bihan D (2003): Looking into the functional architecture of the brain with diffusion MRI. Nat Rev Neurosci 4:469-480.

2. Taylor WD, Hsu E, Krishnan KR, MacFall JR (2004): Diffusion tensor imaging: Background, potential, and utility in psychiatric research. Biol Psychiatry 55:201-207.

3. Basser PJ, Mattiello J, LeBihan D (1994): MR diffusion tensor spectroscopy and imaging. Biophys J 66:259-267.

4. Kochunov P, Thompson PM, Lancaster JL, Bartzokis G, Smith S, Coyle T, et al. (2007): Relationship between white matter fractional anisotropy and other indices of cerebral health in normal aging: Tract-based spatial statistics study of aging. Neuroimage 35:478-487.

5. Song SK, Sun SW, Ramsbottom MJ, Chang C, Russell J, Cross AH (2002): Dysmyelination revealed through MRI as increased radial (but unchanged axial) diffusion of water. Neuroimage 17:1429-1436.

6. Horsfield MA, Jones DK (2002): Applications of diffusion-weighted and diffusion tensor MRI to white matter diseases-a review. NMR Biomed 15:570-577.

7. Adler CM, DelBello MP, Strakowski SM (2006): Brain network dysfunction in bipolar disorder. CNS Spectr 11:312-320; quiz 323-324.

8. Kempton MJ, Geddes JR, Ettinger U, Williams SC, Grasby PM (2008): Meta-analysis, database, and meta-regression of 98 structural imaging studies in bipolar disorder. Arch Gen Psychiatry 65:1017-1032.

9. Pantoni L, Garcia JH (1995): The significance of cerebral white matter abnormalities 100 years after Binswanger's report. A review. Stroke 26: 1293-1301. 
10. Adler CM, Holland SK, Schmithorst V, Wilke M, Weiss KL, Pan H, Strakowski SM (2004): Abnormal frontal white matter tracts in bipolar disorder: A diffusion tensor imaging study. Bipolar Disord 6:197-203.

11. Wang F, Kalmar JH, Edmiston E, Chepenik LG, Bhagwagar Z, Spencer L, et al. (2008): Abnormal corpus callosum integrity in bipolar disorder: A diffusion tensor imaging study. Biol Psychiatry 64:730-733.

12. Wang F, Jackowski M, Kalmar JH, Chepenik LG, Tie K, Qiu M, et al. (2008): Abnormal anterior cingulum integrity in bipolar disorder determined through diffusion tensor imaging. Br J Psychiatry 193:126-129.

13. Sussmann JE, Lymer GK, McKirdy J, Moorhead TW, Maniega SM, Job D, et al. (2009): White matter abnormalities in bipolar disorder and schizophrenia detected using diffusion tensor magnetic resonance imaging. Bipolar Disord 11:11-18.

14. McIntosh AM, Maniega SM, Lymer GK, McKirdy J, Hall J, Sussmann JE, et al. (2008): White matter tractography in bipolar disorder and schizophrenia. Biol Psychiatry 64:1088-1092.

15. Regenold WT, D'Agostino CA, Ramesh N, Hasnain M, Roys S, Gullapalli RP (2006): Diffusion-weighted magnetic resonance imaging of white matter in bipolar disorder: A pilot study. Bipolar Disord 8:188-195.

16. Beyer JL, Taylor WD, MacFall JR, Kuchibhatla M, Payne ME, Provenzale JM, et al. (2005): Cortical white matter microstructural abnormalities in bipolar disorder. Neuropsychopharmacology 30:2225-2229.

17. Haznedar MM, Roversi F, Pallanti S, Baldini-Rossi N, Schnur DB, Licalz EM, et al. (2005): Fronto-thalamo-striatal gray and white matter volumes and anisotropy of their connections in bipolar spectrum illnesses. Biol Psychiatry 57:733-742.

18. Yurgelun-Todd DA, Silveri MM, Gruber SA, Rohan ML, Pimentel PJ (2007): White matter abnormalities observed in bipolar disorder: A diffusion tensor imaging study. Bipolar Disord 9:504-512.

19. Houenou J, Wessa M, Douaud G, Leboyer M, Chanraud S, Perrin M, et al. (2007): Increased white matter connectivity in euthymic bipolar patients: Diffusion tensor tractography between the subgenual cingulate and the amygdalo-hippocampal complex. Mol Psychiatry 12:1001-1010.

20. Bruno S, Cercignani M, Ron MA (2008): White matter abnormalities in bipolar disorder: A voxel-based diffusion tensor imaging study. Bipolar Disord 10:460-468.

21. Chaddock CA, Barker GJ, Marshall N, Schulze K, Hall MH, Fern A, et al. (2009): White matter microstructural impairments and genetic liability to familial bipolar I disorder. Br J Psychiatry 194:527-534.

22. Zanetti MV, Jackowski MP, Versace A, Almeida JR, Hassel S, Duran FL, et al. (2009): State-dependent microstructural white matter changes in bipolar I depression. Eur Arch Psychiatry Clin Neurosci 259:316-328.

23. Mahon K, Wu J, Malhotra AK, Burdick KE, DeRosse P, Ardekani BA, Szeszko PR (2009): A voxel-based diffusion tensor imaging study of white matter in bipolar disorder. Neuropsychopharmacology 34:1590-1600.

24. Smith SM, Jenkinson $M$, Johansen-Berg $H$, Rueckert $D$, Nichols $T E$, Mackay CE, et al. (2006): Tract-based spatial statistics: Voxelwise analysis of multi-subject diffusion data. Neuroimage 31:1487-1505.

25. Versace A, Almeida JR, Hassel S, Walsh ND, Novelli M, Klein CR, et al. (2008): Elevated left and reduced right orbitomedial prefrontal fractional anisotropy in adults with bipolar disorder revealed by tract-based spatial statistics. Arch Gen Psychiatry 65:1041-1052.

26. Wessa M, Houenou J, Leboyer M, Chanraud S, Poupon C, Martinot JL, Paillère-Martinot ML (2009): Microstructural white matter changes in euthymic bipolar patients: A whole-brain diffusion tensor imaging study. Bipolar Disord 11:504-514.

27. Hurley RA, Taber KH (2008): Windows to the Brain: Insights from Neuroimaging. Washington, DC: American Psychiatric Publishing.

28. Jenkinson M, Bannister P, Brady M, Smith S (2002): Improved optimization for the robust and accurate linear registration and motion correc tion of brain images. Neuroimage 17:825-841.

29. Bullmore ET, Suckling J, Overmeyer S, Rabe-Hesketh S, Taylor E, Brammer MJ (1999): Global, voxel, and cluster tests, by theory and permutation, for a difference between two groups of structural MR images of the brain. IEEE Trans Med Imaging 18:32-42.

30. Smith SM, Nichols TE (2009): Threshold-free cluster enhancement: Addressing problems of smoothing, threshold dependence and localisation in cluster inference. Neuroimage 44:83-98.

31. Nichols TE, Holmes AP (2002): Nonparametric permutation tests for functional neuroimaging: A primer with examples. Hum Brain Mapp $15: 1-25$.
32. Faul F, Erdfelder E, Lang AG, Buchner A (2007): G*Power 3: A flexible statistical power analysis program for the social, behavioral, and biomedical sciences. Behav Res Methods 39:175-191.

33. Rich BA, Fromm SJ, Berghorst LH, Dickstein DP, Brotman MA, Pine DS, Leibenluft $E$ (2008): Neural connectivity in children with bipolar disorder: Impairment in the face emotion processing circuit. J Child Psychol Psychiatry 49:88-96.

34. Arnone D, Mclntosh AM, Chandra P, Ebmeier KP (2008): Meta-analysis of magnetic resonance imaging studies of the corpus callosum in bipolar disorder. Acta Psychiatr Scand 118:357-362.

35. Brambilla P, Bellani M, Yeh PH, Soares JC (2009): Myelination in bipolar patients and the effects of mood stabilizers on brain anatomy. Curr Pharm Des 15:2632-2636.

36. Drevets WC, Price JL, Furey ML (2008): Brain structural and functional abnormalities in mood disorders: Implications for neurocircuitry models of depression. Brain Struct Funct 213:93-118.

37. Price JL, Drevets WC (2010): Neurocircuitry of mood disorders. Neuropsychopharmacology 35:192-216.

38. Barnea-Goraly N, Chang KD, Karchemskiy A, Howe ME, Reiss AL (2009): Limbic and corpus callosum aberrations in adolescents with bipolar disorder: A tract-based spatial statistics analysis. Biol Psychiatry 66:238-244.

39. Hoeft F, Barnea-Goraly N, Haas BW, Golarai G, Ng D, Mills D, et al. (2007): More is not always better: Increased fractional anisotropy of superior longitudinal fasciculus associated with poor visuospatial abilities in Williams syndrome. J Neurosci 27:11960-11965.

40. Bearden CE, Hoffman KM, Cannon TD (2001): The neuropsychology and neuroanatomy of bipolar affective disorder: A critical review. Bipolar Disord 3:106-150; discussion:151-153.

41. Lyoo IK, Dager SR, Kim JE, Yoon SJ, Friedman SD, Dunner DL, Renshaw PF (2010): Lithium-induced gray matter volume increase as a neural correlate of treatment response in bipolar disorder: A longitudinal brain imaging study. Neuropsychopharmacology 35:1743-1750.

42. Moore GJ, Cortese BM, Glitz DA, Zajac-Benitez C, Quiroz JA, Uhde TW, et al. (2009): A longitudinal study of the effects of lithium treatment on prefrontal and subgenual prefrontal gray matter volume in treatmentresponsive bipolar disorder patients. J Clin Psychiatry 70:699-705

43. McQuillin A, Rizig M, Gurling HM (2007): A microarray gene expression study of the molecular pharmacology of lithium carbonate on mouse brain mRNA to understand the neurobiology of mood stabilization and treatment of bipolar affective disorder. Pharmacogenet Genomics 17:605-617.

44. Dill J, Wang H, Zhou F, Li S (2008): Inactivation of glycogen synthase kinase 3 promotes axonal growth and recovery in the CNS. $J$ Neurosci 28:8914-8928.

45. Schifitto G, Zhong J, Gill D, Peterson DR, Gaugh MD, Zhu T, et al. (2009): Lithium therapy for human immunodeficiency virus type 1-associated neurocognitive impairment. J Neurovirol 15:176-186.

46. Pierpaoli C, Barnett A, Pajevic S, Chen R, Penix LR, Virta A, Basser P (2001): Water diffusion changes in Wallerian degeneration and their dependence on white matter architecture. Neuroimage 13:1174-1185.

47. Rose SE, Hatzigeorgiou X, Strudwick MW, Durbridge G, Davies PS, Colditz PB (2008): Altered white matter diffusion anisotropy in normal and preterm infants at term-equivalent age. Magn Reson Med 60:761-767.

48. Chao TC, Chou MC, Yang P, Chung HW, Wu MT (2009): Effects of interpolation methods in spatial normalization of diffusion tensor imaging data on group comparison of fractional anisotropy. Magn Reson Imaging 27:681-690.

49. Frazier JA, Breeze JL, Papadimitriou G, Kennedy DN, Hodge SM, Moore CM, et al. (2007): White matter abnormalities in children with and at risk for bipolar disorder. Bipolar Disord 9:799-809.

50. Pavuluri MN, Yang S, Kamineni K, Passarotti AM, Srinivasan G, Harral EM, et al. (2009): Diffusion tensor imaging study of white matter fiber tracts in pediatric bipolar disorder and attention-deficit/hyperactivity disorder. Biol Psychiatry 65:586-593.

51. Adler CM, Adams J, DelBello MP, Holland SK, Schmithorst V, Levine A, et al. (2006): Evidence of white matter pathology in bipolar disorder adolescents experiencing their first episode of mania: A diffusion tensor imaging study. Am J Psychiatry 163:322-324.

52. Kafantaris V, Kingsley P, Ardekani B, Saito E, Lencz T, Lim K, Szeszko P (2009): Lower orbital frontal white matter integrity in adolescents with bipolar I disorder. J Am Acad Child Adolesc Psychiatry 48:79-86.

53. Alexander AL, Lee JE, Lazar M, Field AS (2007): Diffusion tensor imaging of the brain. Neurotherapeutics 4:316-329. 
54. Caetano SC, Silveira CM, Kaur S, Nicoletti M, Hatch JP, Brambilla P, et al (2008): Abnormal corpus callosum myelination in pediatric bipolar patients. J Affect Disord 108:297-301.

55. Choi J, Jeong B, Rohan ML, Polcari AM, Teicher MH (2009): Preliminary evidence for white matter tract abnormalities in young adults exposed to parental verbal abuse. Biol Psychiatry 65:227-234.

56. Tkachev D, Mimmack ML, Ryan MM, Wayland M, Freeman T, Jones PB, et al. (2003): Oligodendrocyte dysfunction in schizophrenia and bipolar disorder. Lancet 362:798-805.

57. Uranova NA, Vostrikov VM, Orlovskaya DD, Rachmanova VI (2004): Oligodendroglial density in the prefrontal cortex in schizophrenia and mood disorders: A study from the Stanley Neuropathology Consortium. Schizophr Res 67:269-275.

58. Padmos RC, Hillegers MH, Knijff EM, Vonk R, Bouvy A, Staal FJ, et al. (2008): A discriminating messenger RNA signature for bipolar disorde formed by an aberrant expression of inflammatory genes in monocytes. Arch Gen Psychiatry 65:395-407.

59. Goldstein BI, Kemp DE, Soczynska JK, McIntyre RS (2009): Inflammation and the phenomenology, pathophysiology, comorbidity, and treatment of bipolar disorder: A systematic review of the literature. $J$ Clin Psychiatry 70:1078-1090.

60. Jeong B, Wible CG, Hashimoto R, Kubicki M (2009): Functional and anatomical connectivity abnormalities in left inferior frontal gyrus in schizophrenia. Hum Brain Mapp 30:4138-4151.

61. Qiu D, Tan LH, Zhou K, Khong PL (2008): Diffusion tensor imaging of normal white matter maturation from late childhood to young adulthood: Voxel-wise evaluation of mean diffusivity, fractional anisotropy, radial and axial diffusivities, and correlation with Reading development. Neuroimage 41:223-232. 